\title{
Efficient Thermal-Light Interconversions Based on Optical Topological Transition in the Metal-Dielectric Multilayered Metamaterials
}

\author{
Jing Zhou, Xi Chen, and L. Jay Guo*
}

Thermal-to-light and light-to-thermal conversions are commonly used in technology fields. In both cases, thermal radiation plays an important role in determining the energy conversion efficiency, and there have been significant efforts to tailor the radiation properties of thermal emitters and absorbers. Typically, photonic crystals, ${ }^{[1-8]}$ metamaterials, ${ }^{[9-14]}$ and multilayered structures ${ }^{[15-17]}$ are utilized to create a tunable band-like radiation for applications such as thermophotovoltaics, coherent IR sources, and radiative cooling. Apart from that, a wideband spectral-selective emitter/absorber is also desired because it can enhance the efficiency of thermal-to-light and light-to-thermal conversions. ${ }^{[18]}$

A prime example of thermal-to-light conversion is the conventional incandescent lighting. Current incandescent lights have very low efficiency because the IR emissivity of a tungsten filament is higher than the visible emissivity, and as a result, most of the energy is radiated as IR light and dissipated as heat. An ideal emitter should be a wideband spectral-selective emitter whose emissivity is unity in the visible range and zero in the IR range so that IR emission is completely inhibited and visible emission is enhanced. Figure 1a sketches a light bulb, where a tungsten filament and an ideal emitter are presented for comparison. As shown by the spectral radiance plots of these two (Figure 1b), an ideal emitter could drastically improve the thermal-light conversion efficiency. In the case of light-to-thermal conversion, concentrating solar power (CSP) is a practical technology that uses curved mirrors to reflect and concentrate sunlight onto receivers that convert the solar power into thermal energy for a heat engine to generate electricity. An ideal receiver should be a good absorber of solar light and a

\author{
Dr. J. Zhou, Prof. L. J. Guo \\ Department of Electrical Engineering and \\ Computer Science \\ University of Michigan \\ Ann Arbor, MI 48109, USA \\ E-mail:guo@umich.edu \\ Dr. J. Zhou \\ National Laboratory for Infrared Physics \\ Shanghai Institute of Technical Physics \\ Chinese Academy of Sciences \\ 500 Yutian Road, Shanghai 200083, P. R. China \\ $X$. Chen, Prof. L. J. Guo \\ Applied Physics \\ University of Michigan \\ Ann Arbor, MI 48109, USA
}

poor radiator of IR light so that most of the incident power is converted into heat and the heat is maintained instead of being wasted through thermal radiation. Figure $1 \mathrm{~b}$ sketches a general concept of a CSP system, where a blackbody receiver and an ideal receiver are presented for comparison. Both of them absorb the solar power greatly, while the ideal receiver emits much less thermal radiation than the blackbody. Since emissivity is equal to absorptivity at thermal equilibrium according to Kirchhoff's law, a spectrum that is unity over the solar range and zero beyond NIR as shown in Figure 1d is ideal for both the emissivity and the absorptivity. Such a wideband spectral-selective emitter/absorber would absorb $89 \%$ of the solar light and emits only $4.3 \%$ of the blackbody radiation at $1000{ }^{\circ} \mathrm{C}$. Therefore, a wideband spectral-selective emitter/absorber would benefit both thermal-light and light-thermal conversions.

Photonic crystals ${ }^{[19-21]}$ and perfect-absorber metamaterials ${ }^{[13,22]}$ have been proposed for wideband spectral-selective emissivity or absorptivity. However, the complexity of the structures hinders practical applications. Actually, the metaldielectric multilayered structure with an optical topological transition $(\mathrm{OTT})^{[23-26]}$ could serve as a wideband spectralselective emitter/absorber. Unlike the case of the multilayered structures that have been studied under the framework of $1 \mathrm{D}$ photonic crystal, ${ }^{[7,27]}$ in our case the thickness of each metal or dielectric layer is much smaller than the wavelengths so the whole structure can be considered as an effective anisotropic medium. ${ }^{[2]}$ The effective permittivity parallel and perpendicular to the multilayers write $\varepsilon_{\|}=f \varepsilon_{\mathrm{m}}+(1-f) \varepsilon_{\mathrm{d}}$ and $\varepsilon_{\perp}=\varepsilon_{\mathrm{m}} \varepsilon_{\mathrm{d}} /\left((1-f) \varepsilon_{\mathrm{m}}+f \varepsilon_{\mathrm{d}}\right)$, respectively. $\varepsilon_{\mathrm{m}}$ is the permittivity of the metal, $\varepsilon_{\mathrm{d}}$ is the permittivity of the dielectric, and $f$ is the fill ratio of the metal. Usually, $\varepsilon_{\|}$undergoes a sign switch at a certain wavelength, which is called the epsilon-near-zero (ENZ) point, while $\varepsilon_{\perp}$ varies slowly around that range. Figure 2a shows the wavelength-dependent $\varepsilon_{\|}$and $\varepsilon_{\perp}$ of an $\mathrm{Au} / \mathrm{Al}_{2} \mathrm{O}_{3}$ multilayered structure with $f=0.15$. For p-polarization, the light modes inside the multilayered structure are determined by $\varepsilon_{\|}$ and $\varepsilon_{\perp}$ together. At the wavelengths larger than the ENZ point $(\approx 710 \mathrm{~nm}), \varepsilon_{\|}<0$ and $\varepsilon_{\perp}>0$ so that the multilayered structure is a hyperbolic metamaterial (HMM). ${ }^{[24]}$ As shown in Figure $2 b$, the isofrequency contour (IFC) at the wavelength of $800 \mathrm{~nm}$ is hyperbolic. Since an HMM only supports high $k$ modes, light from free space (black dashed circle in Figure $2 b$ ) cannot transmit into an HMM but mostly gets reflected, leading to high reflection (Figure $2 \mathrm{~d}$ ). With the decreasing wavelength, $\varepsilon_{\|}$turns into positive around the ENZ point and the multilayered structure undergoes an OTT from an HMM 
(a)

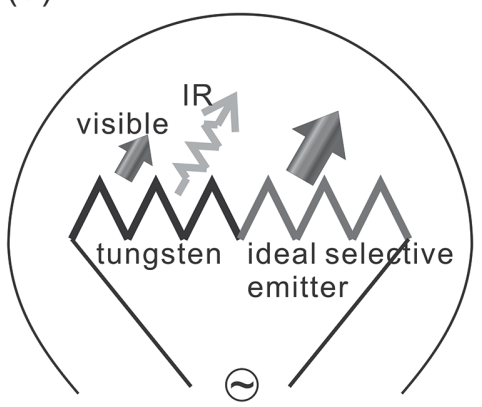

(b)

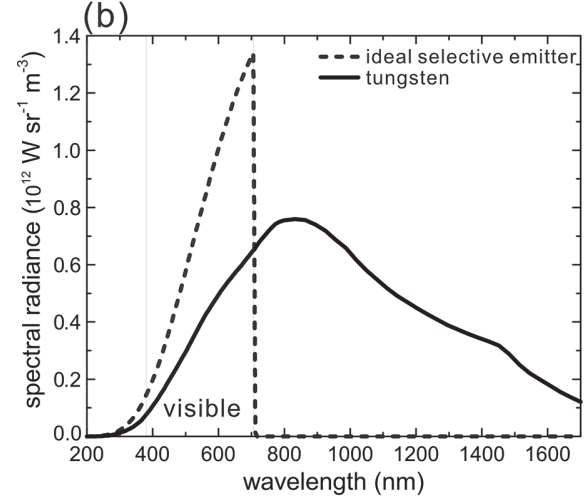

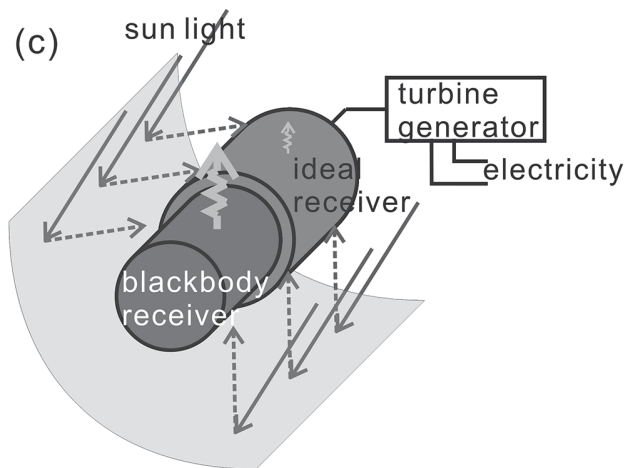

(d)

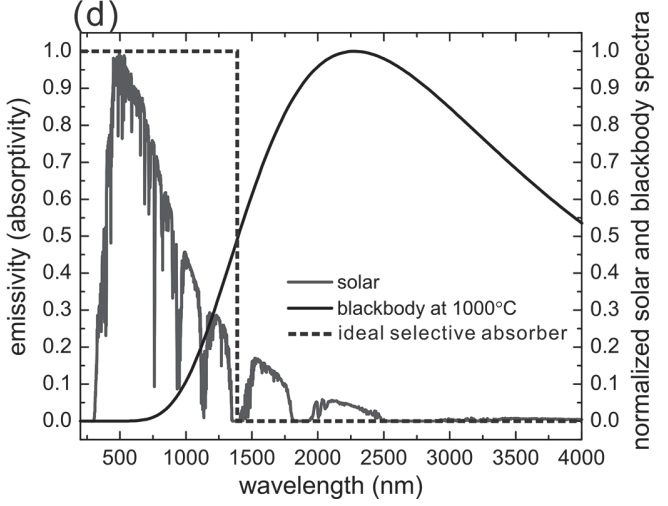

Figure 1. a) Sketch of a light bulb, where a tungsten filament is compared with an ideal emitter that completely inhibits IR radiation, which represents enhanced thermal-light conversion. b) Wavelength-dependent spectral radiance of a tungsten filament and an ideal selective emitter at $3000{ }^{\circ} \mathrm{C}$. The spectral radiance is at the surface normal direction. c) Sketch of a CSP system, where a blackbody receiver is compared with an ideal receiver that absorbs solar light and inhibits infrared thermal radiation. d) Emissivity (absorptivity) of an ideal selective emitter/absorber, the normalized spectra of the solar light (AM1.5) and a $1000^{\circ} \mathrm{C}$ blackbody radiation. The colored version of the figures can be found in the Supporting Information.

to an effective dielectric. Correspondingly, the IFC turns from a hyperboloid into an ellipsoid as shown in Figure 2b. In this case, light can penetrate into the structure and get absorbed, leading to high absorption (Figure 2d). For s-polarized light, only $\varepsilon_{\|}$matters. At the wavelengths longer than the ENZ wavelength, the multilayered structure works as an effective metal so that there is no propagation mode inside the medium (no IFC can be plotted). As a result, the incident light is mostly reflected. Crossing the ENZ point, due to the sign switch of $\varepsilon_{\|}$ the multilayered structure becomes an effective dielectric with a spherical IFC as shown in Figure 2c. Similar to the p-polarized light, the s-polarized light is also highly absorbed in this wavelength range. Therefore, the IFC of a metal-dielectric multilayered structure undergoes a topological transition either from a hyperboloid to an ellipsoid for p-polarized light or from nonexistence to a sphere for s-polarized light. For both polarizations, the multilayered structure turns from a good reflector to a good absorber with the wavelength decreasing across the ENZ point (Figure 2d). By setting the ENZ point at the border between the visible and the IR range through fill ratio tuning (Figure 2e), a wideband spectral-selective emitter/absorber for efficient thermal-light interconversions can be realized. Moreover, the spectra of emissivity/absorptivity for both polarizations are close to each other at moderate incident angles (e.g., $30^{\circ}$ incidence as shown in Figure 2d). Although they start to diverge at large angles (e.g., $60^{\circ}$ incidence as shown in Figure $2 \mathrm{f}$ ), the average over the two polarizations is still close to the normal incidence case. The metamaterial should be sufficiently thick to block the direct light transmission. A significant transmission throughout the metamaterial would cause less absorption in the visible range and less reflection in the IR range so that the performance is degraded. At a certain wavelength, with the fill factor decreasing across the ENZ point, the metamaterial changes from an effective metal to an effective dielectric so that the penetration power as well as the absorption grows rapidly. With the fill factor further decreasing, although the penetration power is considerable, the absorption decreases due to too much diminishing of metal. As a result, a lot of penetration power transmits throughout the metamaterial.

For thermal applications, noble metals like $\mathrm{Au}$ or $\mathrm{Ag}$ are not proper due to the low stability at high temperatures. We choose titanium nitride (TiN) to function as the metal in the multilayered structure. TiN is a refractory material with high-temperature stability and high reflectivity in IR. With optical properties similar to $\mathrm{Au}$, TiN has been proposed for high-temperature applications such as solar/thermophotovoltaics. ${ }^{[28-30]}$ The ENZ wavelength of TiN is around $490 \mathrm{~nm}$. Making TiN into thin slices separated by $\mathrm{SiO}_{2}$ thin film spacers could redshift the ENZ point as shown in Figure 3a. Correspondingly, as shown in Figure $3 \mathrm{~b}$, the absorber/reflector transition wavelength can be tuned by different fill ratios. The absorptivity (emissivity) spectra in Figure 3b are for normal incidence. Since the case at a moderate incident angle is close to that at normal incidence for both polarizations as shown in Figure 3c, only the normal 
(a)
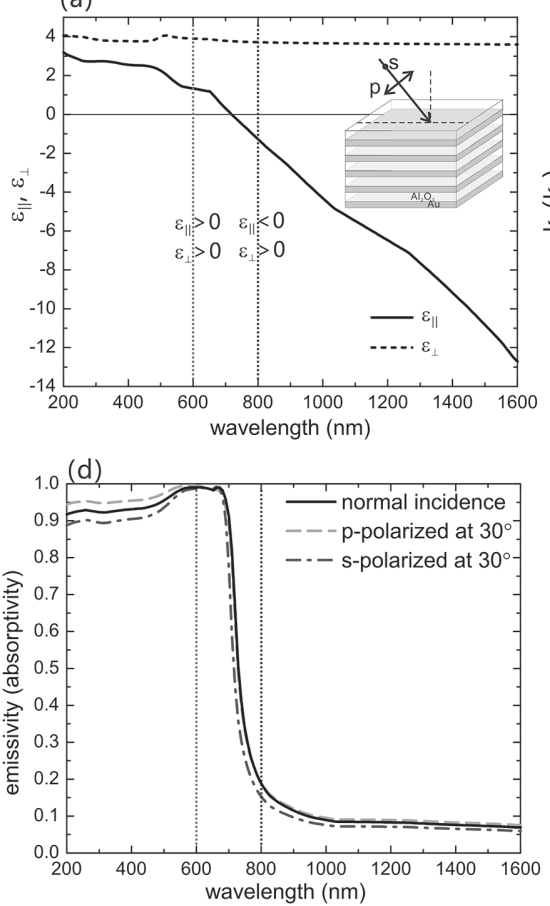

(b)
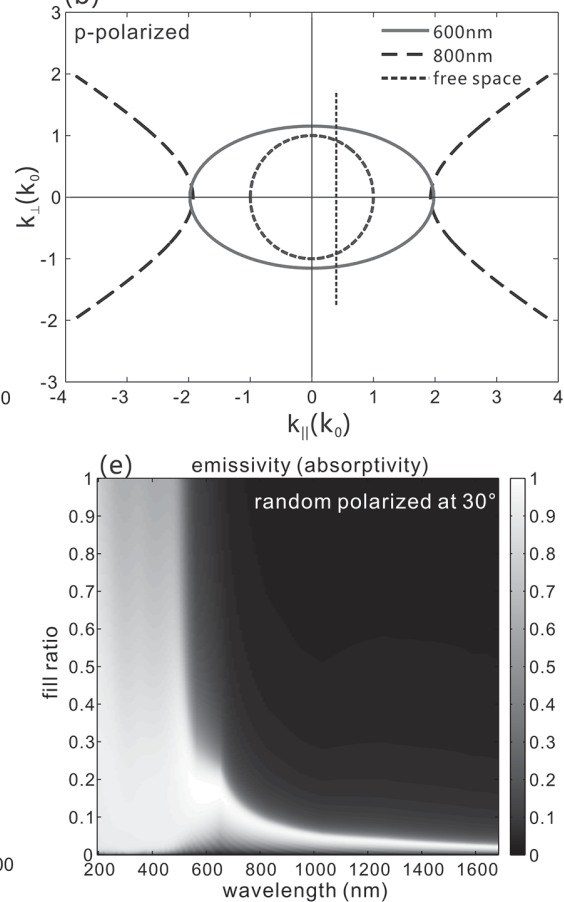
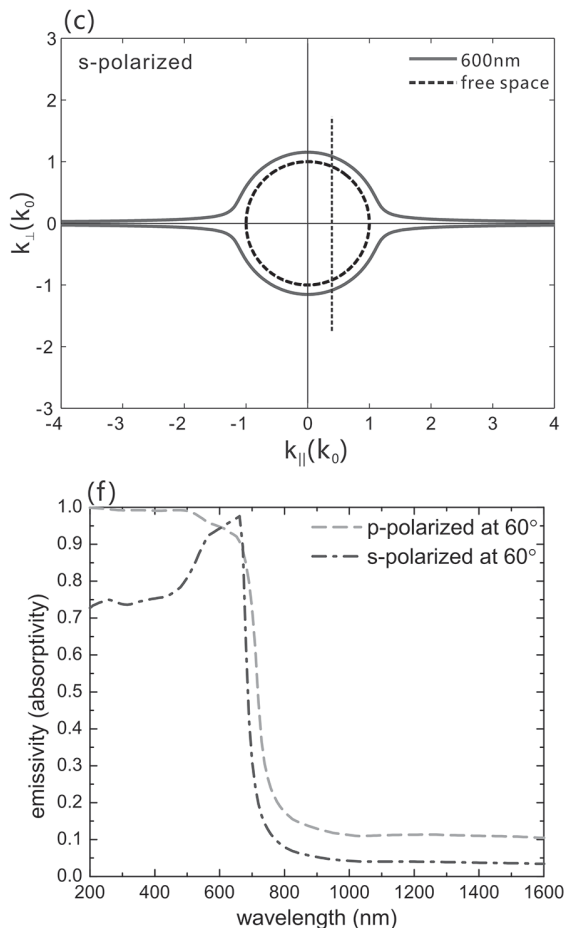

Figure 2. Illustration of optical topological transition in an $\mathrm{Au} / \mathrm{Al}_{2} \mathrm{O}_{3}$ multilayered structure $(f=0.15)$ structure for wideband spectral-selective emission/absorption. a) Wavelength-dependent anisotropic permittivity. b) IFC of p-polarized light in the $\mathrm{Au}_{\mathrm{Al}} \mathrm{Al}_{2} \mathrm{O}_{3}$ multilayered structure at the wavelengths of 600 and $800 \mathrm{~nm}$ and IFC of light in free space. c) IFC of s-polarized light in the $\mathrm{Au} / \mathrm{Al}_{2} \mathrm{O}_{3}$ multilayered structure at the wavelength of $600 \mathrm{~nm}$ and IFC of light in free space. d) Emissivity/absorptivity spectrum of the $\mathrm{Au} / \mathrm{Al}_{2} \mathrm{O}_{3}$ multilayered structure as an effective medium ( $3 \mu \mathrm{m}$ thick) at normal incidence and that at $30^{\circ}$ incidence for both polarizations. e) Emissivity/absorptivity spectra at different fill ratios. Each spectrum is an average over the two polarizations at $30^{\circ}$ incidence. $\mathrm{f}$ ) Emissivity/absorptivity spectra of the $\mathrm{Au} / \mathrm{Al}_{2} \mathrm{O}_{3}$ multilayered structure as an effective medium ( $3 \mu \mathrm{m}$ thick) at $60^{\circ}$ incidence for two polarizations.

incidence spectra are plotted in Figure 3b,d. Figure 3d gives the whole range of the ENZ wavelength that can be shifted by tuning the fill ratio. However, since a low fill ratio of metal leads to a less negative permittivity (Figure 3a), a larger redshift is accompanied by a lower reflectivity in IR. Therefore, the fill ratio needs to be carefully designed for different applications. The plots in Figure 3 are based on the permittivity of the TiN film characterized by spectroscopic ellipsometry. We should mention that TiN is not the only choice. Any material meeting the criteria (1) high-temperature stability and durability, (2) ENZ point in the visible range and close to IR, (3) high IR reflectivity could constitute a metamaterial with OTT for the spectral-selective emitter/absorber application.

The $\mathrm{TiN} / \mathrm{SiO}_{2}$ multilayered structure was fabricated by sputter deposition. The permittivity of a fabricated TiN or $\mathrm{SiO}_{2}$ film was obtained by spectroscopic ellipsometry characterization.

We measured the reflectivity of the multilayered samples at different temperatures by using a custom-made setup that allows the sample to be heated in a vacuum chamber. As shown in Figure 4, the sample is mounted on a heater inside a vacuum chamber pumped down to $8 \times 10^{-7}$ Torr. The heater temperature can be raised up to $800{ }^{\circ} \mathrm{C}$ and can be stabilized at any level below that with a fluctuation smaller than $0.1{ }^{\circ} \mathrm{C}$. The incident light is collected from a broadband source such as a halogen lamp and then guided through a multimode fiber (core size $400 \mu \mathrm{m}$ ). The outgoing light is collimated by a collimator, and then reaches the sample through a view port on the chamber. The reflected light is collected by a spectrometer through another multimode fiber. The viewport window is transparent from visible to NIR $(\approx 3 \mu \mathrm{m})$.

The fabricated $\mathrm{TiN} / \mathrm{SiO}_{2}$ multilayered sample with a golden appearance is shown in Figure 5a. The substrate sample (300 nm oxide on p-type Si) is used as a reference. The crosssectional view of the multilayered sample shows 5 cycles of TiN $(30 \mathrm{~nm}) / \mathrm{SiO}_{2}(20 \mathrm{~nm})$ bilayer films. The reflection measurement results are represented by the (1 - reflectivity) spectra in Figure 5c. Compared to the substrate control sample, the $\mathrm{TiN} / \mathrm{SiO}_{2}$ multilayered structure absorbs more light in the visible range and reflects more in the IR range. Since light can hardly transmit through the $\mathrm{TiN} / \mathrm{SiO}_{2}$ multilayered structure, the $(1$ - reflectivity) spectrum equals to the absorptivity or emissivity spectrum. The averaged absorptivity over the visible range is around 0.68 , the maximum absorptivity is above 0.92 . The emissivity at the wavelengths longer than $1 \mu \mathrm{m}$ is lower than 0.12. Simulations (dashed and dotted lines in Figure $5 \mathrm{c}$ ) were based on the permittivities obtained through ellipsometry characterization of a single layer of TiN or $\mathrm{SiO}_{2}$ fabricated in the same condition for the multilayers. In principle, the measurements and the simulations agree with each other. The deviation at the shorter wavelengths is due to the poor response of the detector in the spectrometer. The same multilayered structure $\left(5\right.$ cycles of TiN $(30 \mathrm{~nm}) / \mathrm{SiO}_{2}(20 \mathrm{~nm})$ bilayer films) was also fabricated on a fused silica substrate. 

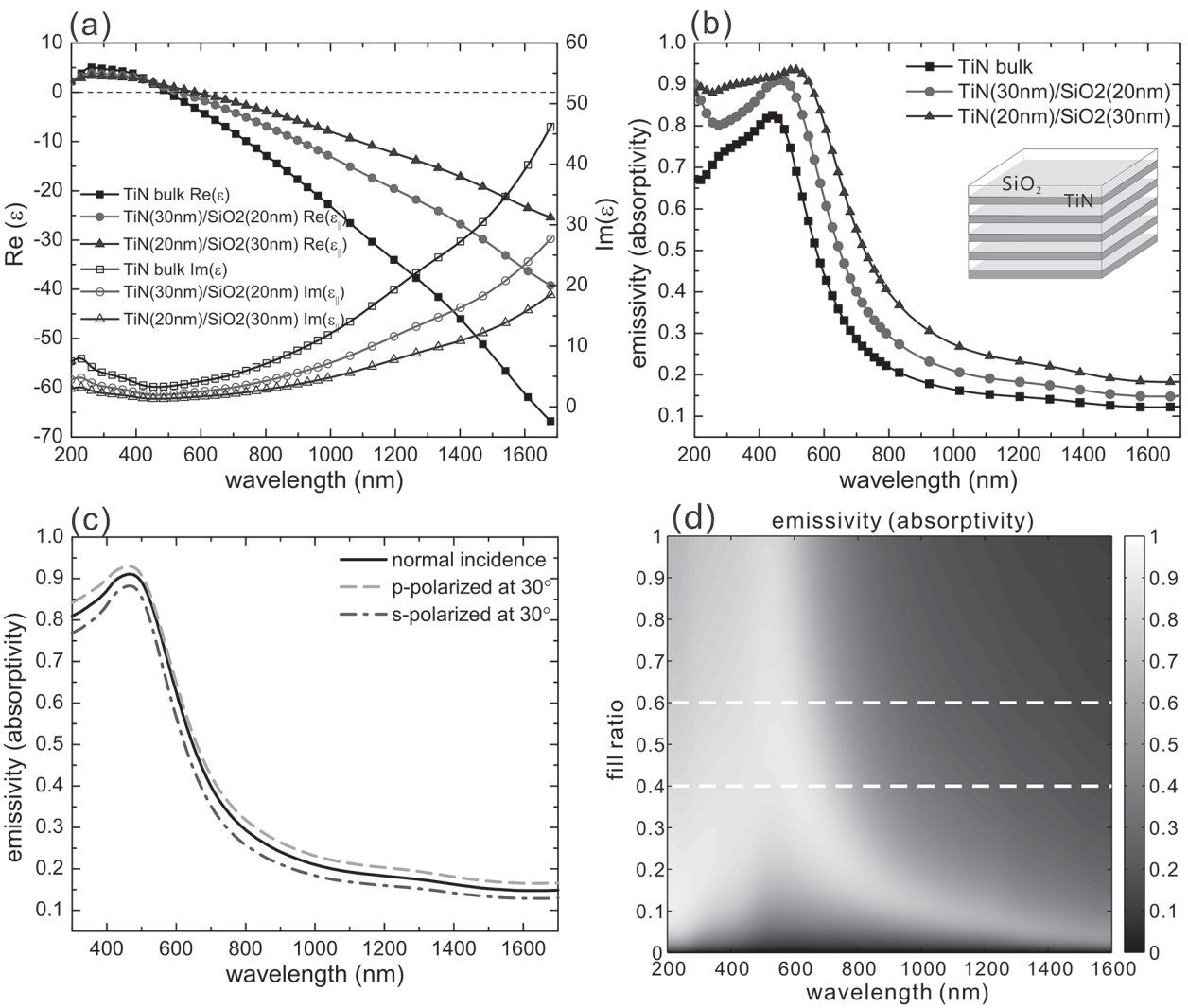

Figure 3. Multilayered structures constituted by $\mathrm{TiN}$ and $\mathrm{SiO}_{2}$. a) Wavelength-dependent effective permittivity $\left(\varepsilon_{\|}\right)$of $\mathrm{TiN}(30 \mathrm{~nm}) / \mathrm{SiO} 2(20 \mathrm{~nm})$ and $\mathrm{TiN}(20 \mathrm{~nm}) / \mathrm{SiO}_{2}(30 \mathrm{~nm})$ as two effective media. The permittivity of TiN is plotted for comparison. b) Emissivity (absorptivity) spectra of two multilayered structures: 5 -cycle $\mathrm{TiN}(30 \mathrm{~nm}) / \mathrm{SiO}_{2}(20 \mathrm{~nm})$ and $\mathrm{TiN}(20 \mathrm{~nm}) / \mathrm{SiO}_{2}(30 \mathrm{~nm})$. The total thickness is $250 \mathrm{~nm}$ for each. The spectrum of a $250 \mathrm{~nm}$ thick TiN bulk is plotted for comparison. c) Emissivity (absorptivity) spectra of 5 -cycle TiN $(30 \mathrm{~nm}) / \mathrm{SiO}_{2}(20 \mathrm{~nm})$ at normal incidence and at $30^{\circ}$ incidence for two polarizations. The spectra in panels (b) and (c) are obtained by simulation of the multilayers instead of an effective medium. d) Emissivity (absorptivity) spectra of the $\mathrm{TiN} / \mathrm{SiO}_{2}$ multilayered structure at different fill ratios. The two dashed lines correspond to the cases of TiN $(30 \mathrm{~nm}) / \mathrm{SiO}_{2}(20 \mathrm{~nm})$ and TiN $(20 \mathrm{~nm}) / \mathrm{SiO}_{2}(30 \mathrm{~nm})$. All plots in panels (a) - (c) are based on the ellipsometry characterizations of the $\mathrm{TiN}$ and the $\mathrm{SiO}_{2}$ we made.

The reflection measurement result agrees with the sample on an oxide-Si substrate. Thus, the influence of the substrate on the performance of the metamaterial is eliminated. Simulation shows that the transmission throughout the 5 cycles of TiN $(30 \mathrm{~nm}) / \mathrm{SiO}_{2}(20 \mathrm{~nm})$ is less than $1.2 \%$ so that the metamaterial is almost isolated from the substrate concerning the optical properties.

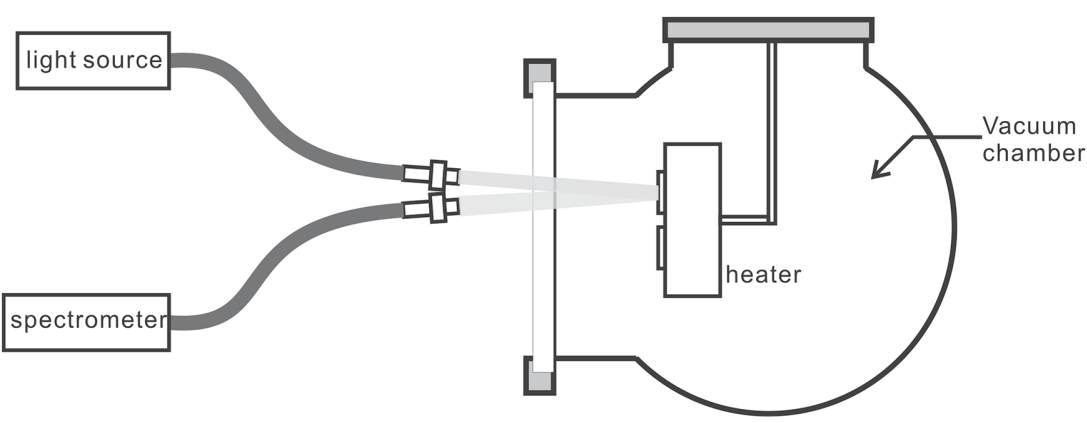

Figure 4. Sketch of the in situ high-temperature reflection measurement system. Samples are mounted on the heater inside a vacuum chamber. A view port on the chamber allows incident light and reflected light to penetrate through.
Since the sample needs to work at a high temperature for the intended energy conversion applications, we studied the optical properties at elevated temperatures. The inset of Figure 6 shows the photograph of the $\mathrm{TiN} / \mathrm{SiO}_{2}$ multilayered sample as well as the reference sample at $750{ }^{\circ} \mathrm{C}$ on the heater. The reflection measurement of the multilayered structure was plotted in Figure 6. The performance of the $\mathrm{TiN} / \mathrm{SiO} \mathrm{O}_{2}$ multilayered structure degrades with the increasing temperature in terms of the decreasing absorptivity in the visible range and the increasing emissivity in the IR range. This can be attributed to the electron-phonon interaction that increases the damping of the electron movement. When the sample cools down, the performance restores. Most of the previous studies on spectral-selective coatings for light-to-thermal conversion performed reflectivity measurement after high-temperature annealing process only to show the thermal stability of the sample. However, an in situ reflectivity measurement at a high temperature was rarely reported, but 

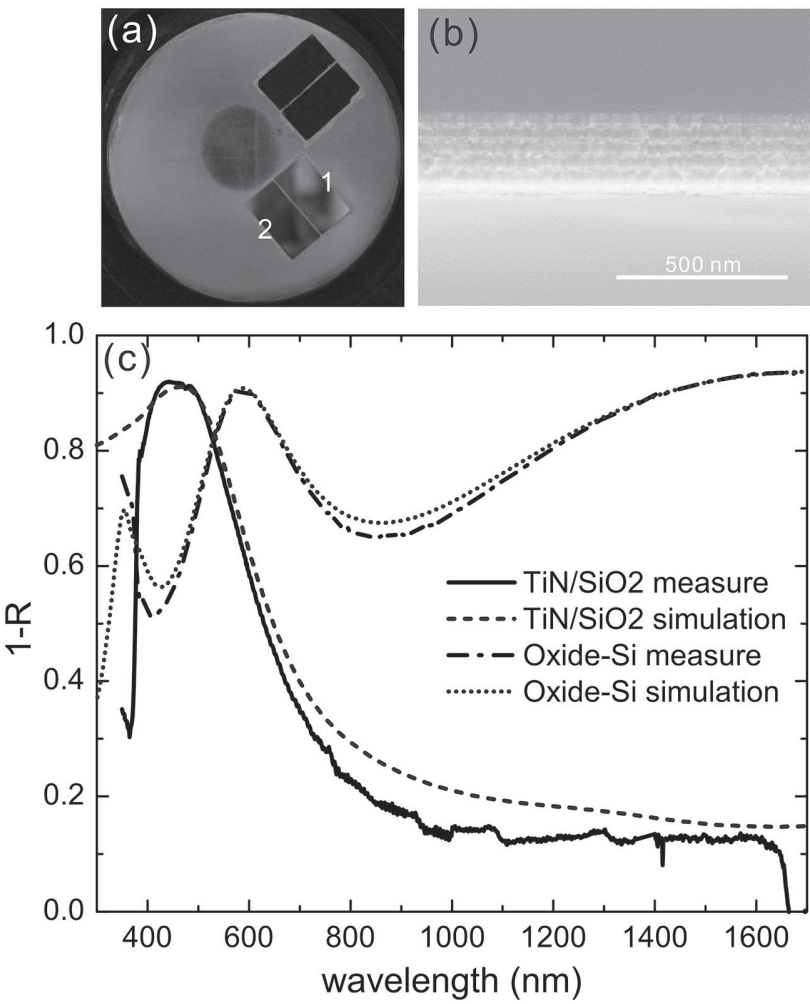

Figure 5. a) Photograph of the 5 -cycle $\mathrm{TiN}(30 \mathrm{~nm}) / \mathrm{SiO}_{2}(20 \mathrm{~nm}) \mathrm{mul}$ tilayers (sample 1) and the substrate ( $300 \mathrm{~nm}$ oxide on a p-type Si) as a reference (sample 2). b) Cross-sectional SEM image of the 5-cycle TiN $(30 \mathrm{~nm}) / \mathrm{SiO}_{2}(20 \mathrm{~nm})$ multilayered sample. c) Spectra of 1 - reflectivity of the 5-cycle $\mathrm{TiN}(30 \mathrm{~nm}) / \mathrm{SiO}_{2}(20 \mathrm{~nm})$ multilayered sample and the reference (300 $\mathrm{nm}$ oxide on p-type Si). Simulation results based on ellipsometry characterization of $\mathrm{TiN}$ or $\mathrm{SiO}_{2}$ single layer are plotted for comparison.

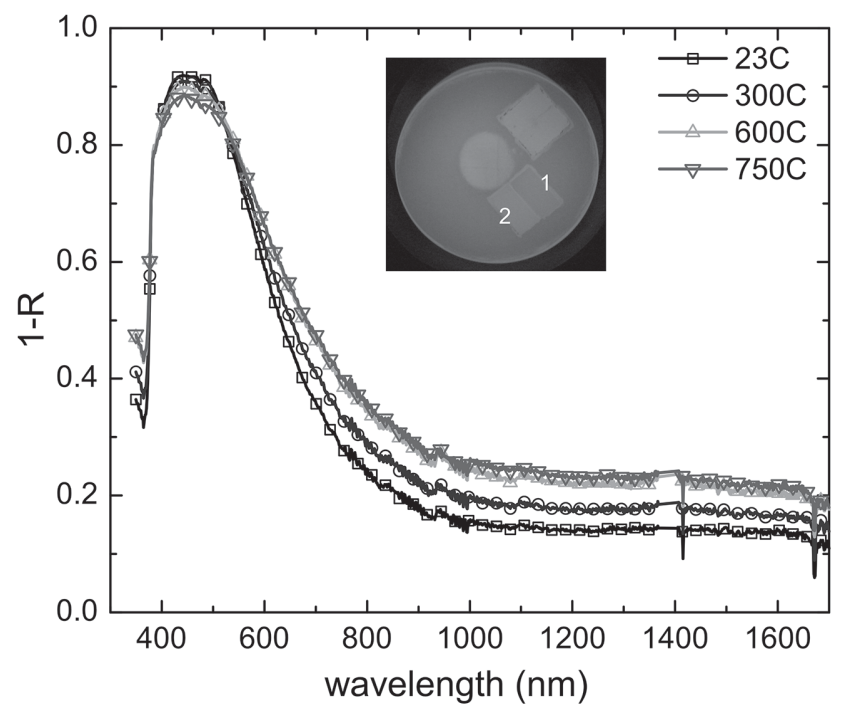

Figure 6. Spectra of 1 - reflectivity of the 5 -cycle $\mathrm{TiN}(30 \mathrm{~nm}) / \mathrm{SiO}_{2}$ $(20 \mathrm{~nm})$ multilayered sample at variant temperatures. The inset shows the 5 -cycle TiN $(30 \mathrm{~nm}) / \mathrm{SiO}_{2}(20 \mathrm{~nm})$ multilayers (sample 1) and the substrate $(300 \mathrm{~nm}$ oxide on a $\mathrm{p}$-type $\mathrm{Si}$ ) as a reference (sample 2) at $750^{\circ} \mathrm{C}$.

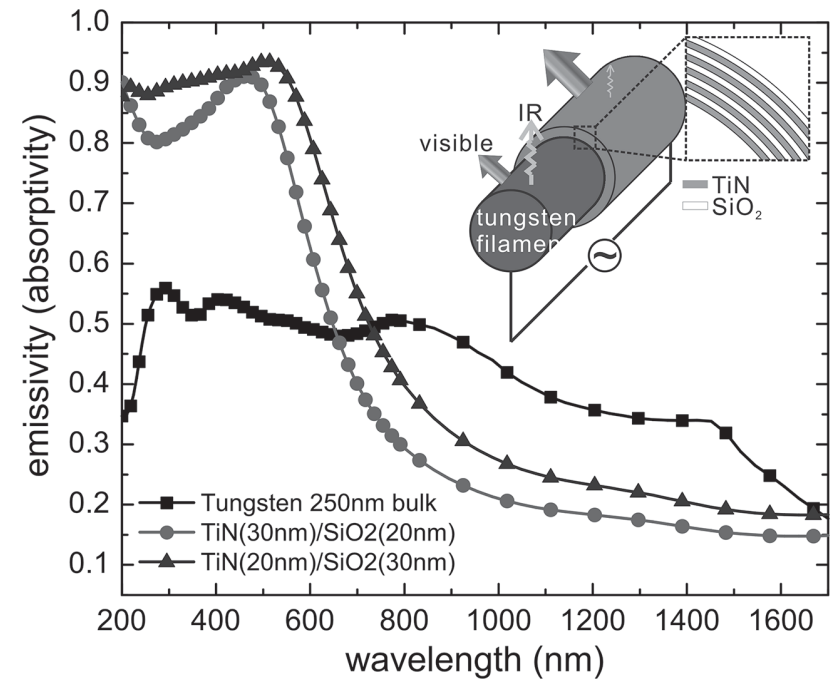

Figure 7. Emissivity of tungsten and two multilayered structures: 5-cycle TiN $(30 \mathrm{~nm}) / \mathrm{SiO}_{2}(20 \mathrm{~nm}), 5$-cycle TiN $(20 \mathrm{~nm}) / \mathrm{SiO}_{2}(30 \mathrm{~nm})$. The inset sketches a bare tungsten filament, partially coated with the multilayered structures. The coated part is expected to behave like a spectral-selective emitter.

is absolutely necessary as material properties could change at elevated temperatures. Since we obtained the temperaturedependent emissivity/absorptivity spectrum through the in situ reflectivity measurement, the performance of such a spectralselective coating can be more accurately estimated for applications at different temperatures.

As stated, a typical thermal-to-light conversion technique is incandescent lighting. A tungsten filament emits a large portion of its thermal energy in the IR instead of the visible range due to the unfavorable emissivity spectrum (Figure 7). If the tungsten filament is coated with the $\mathrm{TiN} / \mathrm{SiO} \mathrm{O}_{2}$ multilayered structure, the thermal emission contains more visible light and less IR light. The ratio of the visible emission to the IR emission could be two to three times higher than that for a bare tungsten filament (Table 1).

The application of the multilayered structures in a practical light-thermal conversion technique like CSP is explored. Since a higher temperature of the engine leads to a higher efficiency according to Carnot's theorem, a high-temperature receiver is pursued. By using fluoride salts as the heat transfer medium, the receiver temperature could be pushed up to $700{ }^{\circ} \mathrm{C}-1000{ }^{\circ} \mathrm{C} \cdot{ }^{[31,32]}$ At such a high temperature, radiation becomes a big waste of the thermal energy. If the CSP receiver is coated with a metal-dielectric multilayered structure such as a 8-cycle TiN $(15 \mathrm{~nm}) / \mathrm{SiO}_{2}(35 \mathrm{~nm})$ as shown in Figure 8 , the thermal radiation at $1000{ }^{\circ} \mathrm{C}$ is reduced to $19 \%$ of the blackbody radiation (estimated in the range from $200 \mathrm{~nm}$ to $4 \mu \mathrm{m}$ ) and the absorption of the solar power is $65 \%$. The emissivity/ absorptivity spectrum of the 8-cycle $\mathrm{TiN}(15 \mathrm{~nm}) / \mathrm{SiO}_{2}(35 \mathrm{~nm})$ multilayered structure together with the normalized solar and blackbody radiation spectra is presented in Figure 8 . The spectrum of the 8-cycle $\mathrm{TiN}(15 \mathrm{~nm}) / \mathrm{SiO}_{2}(35 \mathrm{~nm})$ is a simulation result based on the ellipsometry-characterized permittivity that is extended to mid-IR by fitting with a Drude-Lorentz model. If the TiN reported in ref. ${ }^{[28]}$ (with lower loss) is used to compose the multilayered structure, the absorption of the solar power 
Table 1. Ratio of the visible emission to the near IR emission for a tungsten filament and two multilayered structures at $3000^{\circ} \mathrm{C}$.

\begin{tabular}{lccc}
\hline & Emission in visible $(390-700 \mathrm{~nm})\left[\mathrm{W} \mathrm{sr} \mathrm{m}^{-2}\right]$ & Emission in near IR $\left(700-1700 \mathrm{~nm}^{-1}\left[\mathrm{~W} \mathrm{sr} \mathrm{m}^{-2}\right]\right.$ & Visible/IR ratio \\
\hline Tungsten & $1.21 \times 10^{5}$ & $4.62 \times 10^{5}$ & 0.262 \\
$\mathrm{TiN}(30 \mathrm{~nm}) / \mathrm{SiO}_{2}(20 \mathrm{~nm})$ & $1.57 \times 10^{5}$ & $2.48 \times 10^{5}$ & 0.633 \\
$\mathrm{TiN}(20 \mathrm{~nm}) / \mathrm{SiO}_{2}(30 \mathrm{~nm})$ & $1.90 \times 10^{5}$ & $3.22 \times 10^{5}$ & 0.590 \\
\hline
\end{tabular}

could be enhanced to $67.2 \%$ and the thermal emission can be further suppressed to $16.6 \%$ of the blackbody radiation. The performance could be optimized further by carefully designing the thickness of each layer with the help of some photonic optimization methods. ${ }^{[33]}$ Compared to other spectral-selective coatings, the metal-dielectric multilayered structure has a better suppression of the near IR ( $\leq 2 \mu \mathrm{m})$ emission. At the wavelength of $2 \mu \mathrm{m}$, the emissivity of a typical cermet-based spectral-selective coating is around $50 \%-60 \%,{ }^{[34-36]}$ while the emissivity of the metal-dielectric multilayered structure could be lower than $20 \%$. In addition, the multilayered structures constituted by refractory materials (such as TiN) are more stable at high temperatures. Further, they should have more compact sizes because they do not need additional antireflection coatings or IR reflectors. These advantages indicate a potential application of the metal-dielectric multilayered structure in a high-temperature CPS system.

In conclusion, the metal-dielectric multilayered metamaterials with OTT are proposed to work as wideband spectral-selective emitters/absorbers for efficient thermal-light interconversions. Wideband spectral-selective emitters/absorbers, which are good reflectors for IR light and good absorbers/emitters for visible light, can enhance the thermal energy conversion to visible light and suppress thermal energy waste by radiation. Upon a wavelength decrease across the ENZ point, the metal-dielectric multilayered structure either turns from an HMM to an effective dielectric for p-polarized light or turns from an effective metal to an effective dielectric for s-polarized light. For both polarizations,

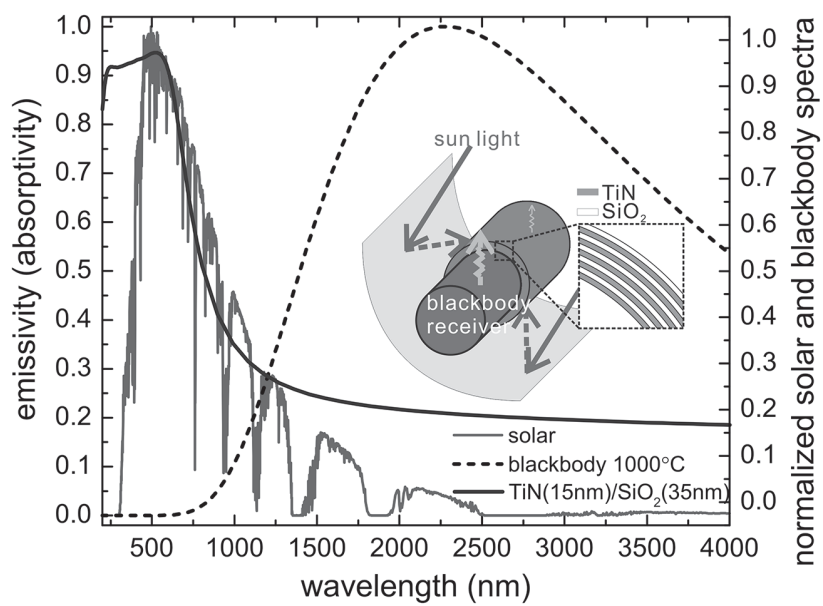

Figure 8. Simulated emissivity (absorptivity) of an 8-cycle TiN (15 nm)/ $\mathrm{SiO}_{2}(35 \mathrm{~nm})$ multilayered structure based on the measured permittivity of our TiN. The normalized spectra of the solar light (AM1.5) and the blackbody radiation at $1000{ }^{\circ} \mathrm{C}$ are shown, respectively. The inset sketches a blackbody receiver, partially coated with the 8 -cycle $\mathrm{TiN}(15 \mathrm{~nm}) / \mathrm{SiO}_{2}$ $(35 \mathrm{~nm})$ multilayered structure. The coated part is expected to behave like a spectral-selective absorber. due to the topological change in IFC, a transition from a good reflector to a good absorber/emitter occurs at the ENZ point. Thus, if we set the ENZ point at the border between the visible and the IR range by tuning the fill ratio, a wideband spectralselective absorber/emitter can be achieved. We fabricated a multilayered structure with $\mathrm{TiN}$ and $\mathrm{SiO}_{2}$. Optical reflection measurements were performed at various temperatures up to $750{ }^{\circ} \mathrm{C}$. The measurement at room temperature agrees with our expectation. The performance degradation at the elevated temperatures is attributed to the enlarged damping of the electron movement due to electron-phonon interaction. Estimations based on our measurements show that the $\mathrm{TiN} / \mathrm{SiO}_{2}$ multilayered structure as a filament coating could enhance the visible/IR emission ratio two- to threefold during incandescent lighting. Working as a CSP receiver at $1000{ }^{\circ} \mathrm{C}$, the $\mathrm{TiN} / \mathrm{SiO}_{2}$ multilayered structure could suppress the thermal radiation to $19 \%$ while still absorbing $65 \%$ of the solar power.

\section{Experimental Section}

The TiN film was made by reactive direct current (DC) sputter deposition. A pre-pumped chamber was filled with $\mathrm{Ar}$ gas and $\mathrm{N}_{2}$ gas at a certain ratio (10:2 in this case). A titanium (Ti) target was sputtered by $\mathrm{Ar}^{+}$ions at a bias of $300 \mathrm{~W}$. The sputtered Ti atoms reacted with the $\mathrm{N}_{2}$ gas and formed TiN deposited on the substrate. An additional DC bias of $50 \mathrm{~W}$ was applied to energize the Ti atoms for adequate reaction when meeting the $\mathrm{N}_{2}$ molecules in the chamber. The $\mathrm{SiO}_{2}$ film was fabricated by radio-frequency sputter deposition. Both TiN films and $\mathrm{SiO}_{2}$ films were made in the same chamber. The films were deposited on a p-doped Si chip with a $300 \mathrm{~nm}$ thermal oxide layer. The oxide layer enhances the ellipsometry characterization of a TiN film (absorptive) by interference, while it has little influence on the optical characterization of the $\mathrm{TiN} / \mathrm{SiO}_{2}$ multilayered structure (adequately thick) which is opaque in the visible and IR range. After deposition, a postanneal process $\left(10 \mathrm{~min}\right.$ of annealing at $800{ }^{\circ} \mathrm{C}$ in forming gas, i.e., $5 \%$ of $\mathrm{H}_{2}$ in $\mathrm{N}_{2}$ ) was performed to anneal the defects in the deposited TiN films. The annealed sample showed a more negative permittivity and a smaller damping, which was desired for a sharp transition from an absorber to a reflector. Further, it was found that heating the sample during deposition helps to improve the quality of the TiN films greatly.

\section{Acknowledgements}

The authors appreciate the help of Prof. Jamie Phillips for the usage of the vacuum heating system in his laboratory. The authors thank LNF staff members Matthew Oonk, David Sebastian, Brian Armstrong, James Kulman, and Terre Briggs for their assistance. The authors also appreciate the helpful discussion with Dr. Weixi Zhou. This work is supported by the NSF Materials Research Science and Engineering Center (Program DMR 1120923).

Received: November 4, 2015

Revised: December 24, 2015

Published online: February 17, 2016 
[1] J. G. Fleming, S. Y. Lin, I. El-Kady, R. Biswas, K. M. Ho, Nature 2002, $417,52$.

[2] J.-J. Greffet, R. Carminati, K. Joulain, J.-P. Mulet, S. Mainguy, Y. Chen, Nature 2002, 416, 61.

[3] N. Dahan, A. Niv, G. Biener, V. Kleiner, E. Hasman, Appl. Phys. Lett. 2005, 86, 191102

[4] I. Celanovic, D. Perreault, J. Kassakian, Phys. Rev. B: Condens. Matter 2005, 72, 075127

[5] A. Lenert, D. M. Bierman, Y. Nam, W. R. Chan, I. Celanović, M. Soljačić, E. N. Wang, Nat. Nanotechnol. 2014, 9, 126.

[6] V. Rinnerbauer, S. Ndao, Y. X. Yeng, W. R. Chan, J. J. Senkevich, J. D. Joannopoulos, M. Soljacic, I. Celanovic, Energy Environ. Sci. 2012, 5, 8815 .

[7] A. Narayanaswamy, G. Chen, Phys. Rev. B: Condens. Matter 2004, 70, 125101

[8] B. J. Lee, C. J. Fu, Z. M. Zhang, Appl. Phys. Lett. 2005, 87, 071904.

[9] I. Puscasu, W. L. Schaich, Appl. Phys. Lett. 2008, 92, 233102.

[10] H. T. Miyazaki, K. Ikeda, T. Kasaya, K. Yamamoto, Y. Inoue, K. Fujimura, T. Kanakugi, M. Okada, K. Hatade, S. Kitagawa, Appl. Phys. Lett. 2008, 92, 141114.

[11] K. Ikeda, H. T. Miyazaki, T. Kasaya, K. Yamamoto, Y. Inoue, K. Fujimura, T. Kanakugi, M. Okada, K. Hatade, S. Kitagawa, Appl. Phys. Lett. 2008, 92, 021117.

[12] J. A. Mason, S. Smith, D. Wasserman, Appl. Phys. Lett. 2011, 98, 241105

[13] X. Liu, T. Tyler, T. Starr, A. F. Starr, N. M. Jokerst, W. J. Padilla, Phys. Rev. Lett. 2011, 107, 045901

[14] L. P. Wang, Z. M. Zhang, Appl. Phys. Lett. 2012, 100, 063902

[15] A. P. Raman, M. A. Anoma, L. Zhu, E. Rephaeli, S. Fan, Nature $2014,515,540$

[16] C. J. Fu, Z. M. Zhang, Opt. Lett. 2005, 30, 1873.

[17] J. Drevillon, K. Joulain, P. Ben-Abdallah, E. Nefzaoui, J. Appl. Phys. 2011, 109, 034315.

[18] J. Zhou, X. Chen, L. J. Guo, presented at CLEO:2015, San Jose, CA, May 2015
[19] G. Veronis, R. W. Dutton, S. Fan, J. Appl. Phys. 2005, 97, 093104

[20] I. Celanovic, N. Jovanovic, J. Kassakian, Appl. Phys. Lett. 2008, 92, 193101.

[21] E. Rephaeli, A. Raman, S. Fan, Nano Lett. 2013, 13, 1457.

[22] H. Wang, L. P. Wang, Opt. Express 2013, 21, A1078.

[23] H. N. S. Krishnamoorthy, Z. Jacob, E. Narimanov, I. Kretzschmar V. M. Menon, Science 2012, 336, 205.

[24] C. L. Cortes, W. Newman, S. Molesky, Z. Jacob, J. Opt. 2012, 14 063001.

[25] Y. Guo, C. L. Cortes, S. Molesky, Z. Jacob, Appl. Phys. Lett. 2012, $101,131106$.

[26] Y. Guo, Z. Jacob, Opt. Express 2013, 21, 15014.

[27] W.-X. Zhou, Y. Shen, E.-T. Hu, Y. Zhao, M.-Y. Sheng, Y.-X. Zheng, S.-Y. Wang, Y.-P. Lee, C.-Z. Wang, D. W. Lynch, L.-Y. Chen, Opt. Express 2012, 20, 28953.

[28] G. V. Naik, J. L. Schroeder, X. Ni, A. V. Kildishev, T. D. Sands, A. Boltasseva, Opt. Mater. Express 2012, 2, 478.

[29] U. Guler, W. Li, A. Boltasseva, A. Kildishev, V. M. Shalaev, presented at CLEO:2014, San Jose, CA, USA, June 2014

[30] J. Liu, U. Guler, W. Li, A. V. Kildishev, A. Boltasseva, V. M. Shalaev presented at CLEO:2014, San Jose, CA, USA, June 2014

[31] C. K. Ho, B. D. Iverson, Renewable Sustainable Energy Rev. 2014, 29, 835.

[32] C. W. Forsberg, P. F. Peterson, H. Zhao, J. Sol. Energy Eng. 2007, $129,141$.

[33] N. P. Sergeant, O. Pincon, M. Agrawal, P. Peumans, Opt. Express 2009, 17, 22800.

[34] F. Cao, K. McEnaney, G. Chen, Z. Ren, Energy Environ. Sci. 2014, 7, 1615.

[35] Q.-C. Zhang, Sol. Energy Mater. Sol. Cells 2000, 62, 63.

[36] C. E. Kennedy, Review of Mid- to High-Temperature Solar Selective Absorber Materials, National Renewable Energy Laboratory, Technical Report, U.S. Department of Commerce, Springfield, VA 2002 